\title{
ORGANIZACIÓN ETNOPOLÍTICA EN LA REGIÓN METROPOLITANA DE BUENOS AIRES, ARGENTINA
}

\section{ETHNOPOLITICAL ORGANIZATION IN THE METROPOLITAN REGION OF BUENOS AIRES, ARGENTINA}

\section{Juan Manuel Engelman Garreta*}

RESUMEN

\begin{abstract}
El presente trabajo tiene como objetivo describir, a través de diferentes instancias de negociación, las modalidades mediante las cuales las dirigencias indígenas revierten su posición subordinada. Las siguientes reflexiones surgen de la labor etnográfica con diversas comunidades y nucleamientos ${ }^{1}$ indígenas asentados en el municipio de Almirante Brown de la Región Metropolitana de Buenos Aires (RMBA), quienes desde inicios del año 2000 protagonizan un proceso de visibilización y organización etnopolítica local.
\end{abstract}

PALABRAS CLAVE: ARGENTINA * INDÍGENAS URBANOS * ETNICIDAD * POLÍTICAS PÚBLICAS

ABSTRACT

The present paper describes how indigenous leaders revert their subordinate position trough their local and ethno-political practice, according to different negotiation instances. The following reflections arise from the ethnographic work with a group of diverse indigenous communities established in the municipality of Almirante Brown of the Metropolitan Region of Buenos Aires, who since the beginning of the 2000 year have been protagonizing a visibility and local ethno-political organization process.

KEYWORDS: ARGENTINE * URBAN INDIGENOUS * ETHNICITY * PUBLIC POLICIES

Instituto de Ciencias Antropológicas (ICA) de la Facultad de Filosofía y Letras (FFyL) de la Universidad de Buenos Aires, Argentina.

jmengelman@hotmail.com

1 Liliana Tamagno (2014) define la noción de "nucleamiento" para referirse al proceso migratorio de la población indígena y a su capacidad de nuclearse —en la medida que las condiciones materiales lo permitan- en espacios geográficamente diferentes y lejanos a los de origen. 
POBLACIÓN INDÍGENA EN LA REGIÓN METROPOLITANA DE BUENOS AIRES

La organización política de las poblaciones indígenas en las estructuras interculturales supone un abordaje que implica resaltar tanto su carácter resistivo como subordinado, respecto de instancias de administración estatal o en relación con organismos o movimientos sociales. Esa posición de subordinación es, muchas veces, un hecho concreto siendo que constriñe procesos de negociación y reconocimiento. Sin embargo, para poder acentuar ciertas instancias de decisión autonómica de la población indígena, se entiende que su relevancia analítica debe ser matizada. Se trata de reparar en que la organización etnopolítica es el resultado de múltiples vínculos y actores que han reorientado objetivos, modalidades de participación y motivación de las dirigencias indígenas contemporáneas, ya que condensan experiencias específicas e históricas de organización en un tiempo y espacio determinado.
En cuanto a la organización y estructura, se inicia el análisis a través de la presentación de datos demográficos y de las condiciones de vida urbana de la población indígena asentada específicamente en los 24 partidos del Gran Buenos Aires de la RMBA. En segundo lugar, dichos datos se pondrán en diálogo junto con los reclamos que los dirigentes realizan a los gobiernos municipales, provinciales $y$ a diversos organismos institucionales con el objetivo de resaltar aquellas instancias etnopolíticas que marcan la ruptura respecto de los abordajes que consideran a la población indígena como sujetos subordinados. Finalmente, se presenta una breve reflexión.

La denominada "Región Metropolitana de Buenos Aires" está conformada por la Ciudad Autónoma de Buenos Aires (CABA) y los 24 partidos del Gran Buenos Aires (GBA), e incluye un área geográfica mayor definida por un conjunto de partidos contiguos cuyo número varía según los criterios que diferentes organismos o programas estatales eligen para su delimitación (ver mapa 1).

MAPA 1

PARTIDOS Y CORDONES DEL GRAN BUENOS AIRES Y LA REGIÓN METROPOLITANA DE BUENOS AIRES

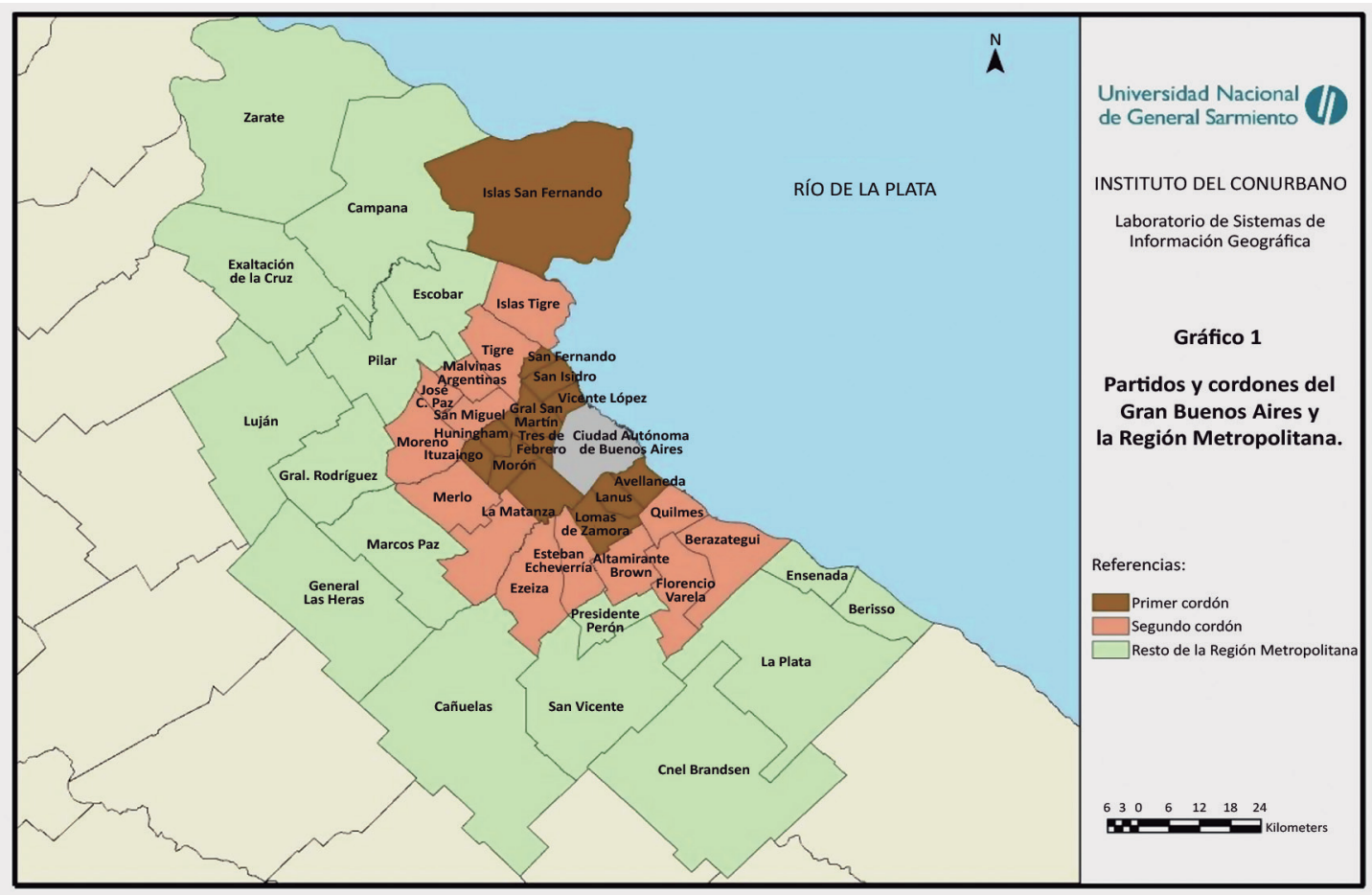

Fuente: $\quad$ Maceira, 2002. 
De acuerdo a un documento publicado por el Instituto Nacional de Estadísticas y Censos (INDEC) en el año 2015, en los 24 partidos del GBA habitan 186640 indígenas que se autoreconocen o descienden de un pueblo originario. Si a este número se le incorpora la cifra de indígenas censados en la CABA, el cual asciende a 61876 , se obtiene como resultado que 248516 indígenas habitan en la RMBA (INDEC, 2015). Ahora bien, si ese número se compara con el total de población indígena de la Argentina (955 032) (INDEC, 2010), se puede afirmar que un $26,02 \%$ de la población indígena nacional habita en la RMBA. Este primer dato muestra que un cuarto de la población indígena vive en una de las zonas con mayor densidad de población de la provincia de Buenos Aires, e incluso del país, lo cual implica un conjunto de reflexiones.
En primer lugar, ese porcentaje refiere acerca de que es irrefutable negar una fuerte presencia indígena en la ciudad, pero también implica irrefutable considerar el impacto efectivo que un conjunto de mecanismos históricos de negación tuvieron al momento de invisibilizar su presencia urbana ${ }^{2}$. En segundo lugar, ese mismo porcentaje permite acentuar una de las características más relevantes que define a las poblaciones indígenas urbanas, una composición heterogénea en términos culturales e históricos ${ }^{3}$. En el caso de los 24 partidos del GBA, la población indígena representa un $19,54 \%$ del total de población indígena nacional, $y$ de acuerdo a la tabla 1 , queda clara su heterogénea composición en la periferia urbana.

$2 \quad$ Alejandro Balazote analiza ciertas representaciones que operaron como sustento en la "construcción de la nación" argentina. Al caracterizar algunas de ellas, sostiene que esas configuraciones de sentido facilitaron la operatoria de mecanismos que delinearon el diseño del formato de relacionamiento del Estado Nacional y los pueblos originarios. Entre estas menciona: "la construcción de opuestos", "la lucha contra el infiel" o "la invisibilización: del desierto a los censos" (Balazote, 2015).

3 Los procesos migratorios no solo han marcado trayectorias diferenciadas en el tejido urbano, sino que dan cuenta de las posibilidades de acceder a los terrenos en función de las diversas condiciones materiales de los lugares de origen, $y$ de las marcadas redes de parentesco y alianza que expresan una organización dinámica en el espacio de recepción. 
TABLA 1

DISTRIBUCIÓN DE LA POBLACIÓN INDÍGENA POR PUEBLO INDÍGENA U ORIGINARIO DE LOS 24 PARTIDOS DEL GRAN BUENOS AIRES

\begin{tabular}{l|c|c}
\hline \multicolumn{1}{c|}{$\begin{array}{c}\text { PUEBLO INDÍGENA } \\
\text { U ORIGINARIO }\end{array}$} & $\begin{array}{c}\text { PORCENTAJE DE PUEBLO } \\
\text { INDÍGENA U ORIGINARIO } \\
\text { EN RELACIÓN AL TOTAL DE } \\
\text { POBLACIÓN DE LA RMBA }\end{array}$ \\
\hline Guaraní & 39571 & $21,20 \%$ \\
Toba & 35544 & $19,04 \%$ \\
Otros & 26914 & $14,42 \%$ \\
Mapuche & 21041 & $11,27 \%$ \\
Quechua & 19630 & $10,51 \%$ \\
Diaguita-Calchaquí & 14269 & $7,64 \%$ \\
Kolla & 6675 & $3,57 \%$ \\
Aymara & 6152 & $3,29 \%$ \\
Pampa & 5694 & $3,05 \%$ \\
Wichí & 5586 & $2,99 \%$ \\
Comechingón & 5564 & $2,98 \%$ \\
\hline TOTAL & 186640 & $100 \%$ \\
\hline
\end{tabular}

Fuente: $\quad$ INDEC, 2015.

En la tabla 1 se aprecia que 5 son los pueblos que se destacan por su fuerte presencia en la región, siendo de mayor a menor cantidad, el Guaraní, el Toba, el Mapuche, el Quechua y el Diaguita-Calchaquí. Entre ellos se concentra casi el $70 \%$ de la población indígena de los 24 partidos del GBA, siendo que el $30 \%$ restante se autoreconoce o desciende de más de 30 pueblos indígenas $\mathrm{u}$ originarios ${ }^{4}$. Otro hecho a destacar y que permite argumentar una presencia indígena desde cierta profundidad histórica en la periferia de la CABA, se condice con la distribución por grupos de edad de la población.

El documento del INDEC distingue tres grupos etarios: el primero es de "0-14 años", el segundo de "15-64 años" y el tercero de "65 años y más". Al primero le corresponde un

$4 \quad$ En un mapa elaborado por el Encuentro Territorial Nacional de Organizaciones Territoriales de Pueblos Originarios (ENOTPO) se ubican 38 pueblos naciones originarias en la Argentina. número de 46991 jóvenes, al segundo 127444 adultos y finalmente al tercero 12205 ancianos $^{5}$. Al retomar el total de población indígena de los 24 partidos del GBA (18 640), fácilmente se puede evidenciar que los 46991 jóvenes censados representan un $25,17 \%$ del total de la población indígena (INDEC, 2015); es decir, un cuarto de la población indígena ha nacido en el ámbito urbano. Si bien, la variable censal de 0 a 14 años restringiría la profundidad histórica a una presencia indígena en la ciudad de una temporalidad similar, el interés remite solamente en destacar su importancia numérica.

$5 \quad$ La existencia de trabajos antropológicos acerca de los jóvenes indígenas urbanos pone en tensión la categorización de la edad como entidad que se define por criterios biológicos. De este modo, lo joven o lo adulto han de ser vistas como clasificaciones sociales que suponen la existencia de un complejo sistema de diferencias, cuya articulación otorga los sentidos y contenidos de cada instancia o fase etaria (Pérez, 2008). 
La existencia de diversos trabajos académicos (Ratier, 1975; Amodio, 1996; Tamagno, 2001 y 2014; Maidana, 2009; Engelman y Weiss, 2015) y testimonios etnográficos ya ubican la mayoría de las oleadas migratorias entre las décadas de 1960 y 1980.

Estos datos se vuelven aún más relevantes si se considera que el 88,6\% (165 432) de los indígenas de la RMBA nació en la Argentina y que de ese total, el 61,1\% (100 705) lo hizo en la provincia de Buenos Aires (INDEC, 2015).

En cuanto a la educación, el documento del INDEC sistematiza información de la población indígena que ha superado los 5 años de edad. Ese número asciende a los 110366 indígenas, de los cuales 12605 no han completado el nivel primario, y 28084 sí lo han hecho. Para el nivel secundario, las cifras son bastante similares, ya que 23619 no lo han completado y 26 874 que sí lo han finalizado. Por último, en el documento se distingue la formación en educación superior.

Los indicadores acerca del trabajo de la población indígena que vive en la periferia de la CABA distinguen entre población económicamente activa de económicamente no activa. Al interior del primer grupo, el documento arroja que de un total de 98682 indígenas, solo 7228 están en situación de desocupación. Ahora bien, si se suma esa cifra con el número de población no económicamente activa (45 053), se encuentra que el $36,8 \%$ de la población no posee un empleo formal, mientras que el $63,2 \%$ restante sí lo posee.

Por último, se puntualiza algunos resultados estadísticos de las condiciones de vida de las viviendas indígenas. En primer lugar, el índice de hacinamiento (2 o más personas que habitan en un mismo cuarto) es del $20,8 \%$ de un total de 73879 hogares, siendo este un porcentaje similar al 22,8\% de viviendas censadas bajo la categoría de "deficitarias". Cabe aclarar que en esta última noción entran todos aquellos hogares que tienen piso de tierra o ladrillo suelto $u$ otro material, asimismo, que no tiene provisión de agua por cañería dentro de la vivienda (INDEC, 2015). En segundo lugar, ello está relacionado a las precarias condiciones de vida que un alto porcentaje de los hogares indígenas $u$ originarios poseen ediliciamente. Se trata de que de ese total de 73879 hogares censados, casi un $40 \%$ no están conectados a la red de gas, por lo que utilizan garrafas, gas de tubo $\mathrm{u}$ otras formas de combustión (INDEC, 2015).

De los datos mencionados, se puede hacer ciertas generalizaciones parciales acerca del acceso y permanencia educativa, trabajo $y$ condiciones de vida de la población indígena que habita en los 24 partidos del GBA. Una de ellas es que 4 de cada 10 indígenas urbanos no ha podido terminar ya sea el nivel primario, secundario o terciario/universitario. Los datos acerca del trabajo permiten sostener que 3 de cada 10 indígenas del GBA se encuentran por fuera de una relación de trabajo formal, y que 2 de cada 10 hogares no solo tienen un alto índice de hacinamiento sino que la higiene y la seguridad de sus habitantes está altamente comprometida. Evidentemente, según los datos estadísticos, más de un tercio de la población indígena urbana y que reside en la periferia de la CABA, se encuentra bajo una situación de vulnerabilidad socioeconómica.

Ahora bien, cabe señalar que la breve presentación de datos estadísticos refiere solamente a un sector de la población indígena censada. Diferenciar entre estimaciones y estudios censales (Trinchero, 2010) se vuelve una herramienta imprescindible para elaborar cierta evaluación cuantitativa. Según Alejandro Balazote (2015), en cada ocasión que se llevó a cabo una medición de la población indígena le correspondió, a grandes rasgos, un motivo subyacente. Los primeros sondeos demográficos fueron durante las campañas de conquista en el siglo XIX y tuvieron por objetivo conocer la información militar de la población indígena. Las mediciones posteriores de la década de 1960 resultaron importantes para dimensionar su condición jurídica. De este modo, es que se interroga acerca de los motivos subyacentes que esconden las estimaciones recientes dado que "en el último censo, la utilización del criterio de autoadscripción permitió reparar en las características socioculturales" (p.47). 
POBLACIÓN INDÍGENA EN LA REGIÓN METROPOLITANA DE BUENOS AIRES. EL CASO DEL MUNICIPIO DE ALMIRANTE BROWN

La RMBA está compuesta por la CABA y 43 unidades administrativas denominadas municipios o "partidos". Su extensión total es de 18 $380 \mathrm{~km}^{2}$ y en esta convive una población total de 12806866 habitantes, de los cuales 248516 se autoreconocen descendientes o pertenecientes a un pueblo originario.

$\mathrm{Al}$ interior de este espacio, se consolidaron diferentes procesos de organización etnopolítica $^{6}$, sobretodo posteriormente al año 1994 , cuando la población indígena fue reconocida como sujeto de derecho gracias a la reforma de la Constitución Nacional ${ }^{7}$. Esto último, sumado al impacto de políticas y programas sociales —desde mediados del año 2000- estimuló y contribuyó a generar dicho escenario.

El presente trabajo reúne las experiencias contemporáneas de un grupo de hombres y mujeres dirigentes indígenas que arribaron al tejido de la RMBA a temprana edad y a mediados de la década de 1960. Estas reflexiones a su vez, surgen de un recorte espacial que se condice con la extensión del municipio de Almirante Brown, el cual se localiza con dirección sur a la CABA.

La delimitación de un área de trabajo responde meramente a una estrategia metodológica dado que tanto las relaciones de las poblaciones indígenas como la de dirigentes, exceden la jurisdicción del municipio. Desde mediados del año 2008, se inicia un trabajo etnográfico junto con familias indígenas migrantes - quienes cuentan con una generación nacida en la ciudad-, procedentes de

6 Los trabajos pioneros de Liliana Tamagno abordan la constitución y organización de un barrio Toba (Qom) en la periferia de la ciudad de La Plata a través de un proyecto de auto-construcción de viviendas otorgado por el Estado (2001). Más recientemente, Carolina Maidana describe los procesos de territorialización de población Toba migrante en la periferia del GBA (2009).

La reforma del texto constitucional del año 1994, permitió introducir algunos instrumentos tales como el art. 75 inc. 17, el cual constituyó un claro avance en el plano jurídico en cuanto a reconocimiento de derechos hacia los pueblos originarios. las provincias de Salta y Chaco del Norte de la Argentina. La composición étnico-identitaria de la población indígena de Almirante Brown es diversa $y$ condensa causas y motivos migratorios muy diferenciados según fueran sus trayectorias históricas y experiencias familiares. $\mathrm{Al}$ inicio del trabajo, se repara en que la heterogeneidad es una de las características que define a la población indígena en la ciudad, aspecto fácilmente reconocible en Almirante Brown.

En el "Consejo Indígena de Almirante Brown" (СIAB) participan dirigentes indígenas de familias migrantes del pueblo Mocoví, Kolla, Qom (Toba), Mapuche, Diaguita-Calchaquí, Tupí-Guaraní y Avá-Guaraní. A simple vista, $y$ en tanto espacio etnopolítico local, el CIAB reúne una complejidad que responde a un proceso común de expulsión territorial. De igual modo, las condiciones de vida urbana -en relación a problemáticas compartidas entre las diferentes familias indígenas- serán canales de cohesión y delimitación de objetivos comunes. Con ello, se entiende que el alto nivel de heterogeneización que define la composición étnico-identitaria de la población indígena en la ciudad no invalida su organización, dado que esta encuentra cause $-y$ está altamente determinado- en procesos históricos vinculados a: 1) la expropiación y violencia territorial sufrida en los lugares de procedencia, 2) trayectorias migratorias articuladas, muchas veces, desde redes de parentesco y de alianza, y 3) a la formación y participación etnopolítica en espacios compartidos de la provincia de Buenos Aires.

El CIAB es una organización que surge a partir de la iniciativa de un grupo de dirigentes indígenas locales. Su composición, formada entre hombres y mujeres, expresa una lucha que buscó inicialmente el reconocimiento de las diversas familias ante el Estado. Este proceso, definido por la obtención de las personerías jurídicas indígenas, fue un eje cohesionador durante los primeros años del 2000. La "personería jurídica" es una figura de reconocimiento estatal que otorga el Instituto Nacional de Asuntos Indígenas (INAI) a través del Registro Nacional de Comunidades Indígenas (Re.Na.Ci), a todas aquellas comunidades que cumplen con un conjunto de requisitos. Entre ellos, según la 
Res. nro. 4811/96, las familias deben presentar un nombre comunitario y ubicación geográfica; una reseña histórica que acredite su origen étnico-cultural; una descripción de sus pautas de organización con mecanismos de designación y remoción de autoridades, así como, una nómina de integrantes y los mecanismos de integración y exclusión de sus miembros.

A continuación en el cuadro 1, se detallan las comunidades reconocidas de las noreconocidas que forman parte del CIAB hasta la actualidad.

CUADRO 1

COMPOSICIÓN DEL "CONSEJO INDÍGENA DE ALMIRANTE BROWN"

\begin{tabular}{|c|c|c|c|c|}
\hline & NOMBRE DE LA COMUNIDAD & PUEBLO & LOCALIDAD & $\begin{array}{l}\text { PERSONERÍA } \\
\text { JURÍDICA }\end{array}$ \\
\hline 1 & "Cacique Hipólito Yumbay" & Tupí-Guaraní & Glew & Posee \\
\hline 2 & "Guaguajni Jall'pa" & Kolla & Glew & Posee \\
\hline 3 & "Migtagan" & Toba & San José & Posee \\
\hline 4 & "Cuibae Toro" & Avá-Guaraní & Glew & Posee \\
\hline 5 & "Nogoyin Ni Nala" & Mocoví & Rafael Calzada & En Trámite \\
\hline 6 & "Cacique Catán” & Mocoví & Longchamps & En Trámite \\
\hline 7 & "Juan Kalfulkurá" & Mapuche & Longchamps & En Trámite \\
\hline
\end{tabular}

Fuente: Elaboración propia.

Si bien, el CIAB es creado formalmente en el año 2007, se puede marcar su inicio a fines de la década de 1990, cuando un conjunto de intereses confluyeron en la necesidad de crear un espacio de participación indígena en el municipio. Entre esos intereses se destacaba la necesidad de reclamar y regularizar territorios urbanos, $y$ delimitar objetivos que permitieran posteriormente presentar un conjunto de demandas específicas.

Es así que los dirigentes convocaron reuniones en sus hogares para debatir procedimientos y para fomentar la visibilización de la población indígena local a través de la realización de "eventos culturales" 8 . Tales eventos reunían a la población indígena en fechas determinadas, en las cuales se realizaban bailes, comidas típicas y donde se usaban los trajes tradicionales de los diversos pueblos. A su vez, esa estrategia de visibilización era acompañada por

$8 \quad$ La noción de "eventos culturales" da cuenta del trabajo político en los barrios desde una perspectiva que prioriza lo étnico-cultural por sobre el interés político partidario. discursos que al tiempo que exigían el reconocimiento de derechos territoriales e identitarios, reclamaban la necesidad de mejorar las condiciones de vida de la población indígena y de los vecinos que acompañaban el proceso de organización. Ese carácter intercultural del espacio de formación etnopolítico en Almirante Brown, encuentra sentido si se considera que las trayectorias de formación de las nuevas generaciones de dirigentes indígenas se han constituido como parte del entramado de la política barrial, y gracias a la reproducción de vínculos asistenciales y paternalistas con el gobierno local. Al respecto, en los primeros años del 2000, una dirigente del pueblo Kolla mencionaba que:

El silencio del indio domesticado en algún momento debía terminar. Así es como comenzó la aventura de darle lugar a esas voces $y$ crear con todas ellas una propuesta política propia para todas las áreas, incluyendo la política partidaria (mujer dirigente Kolla de "Guaguajni Jallpa”, localidad de Glew, comunicación personal, 27/09/2013). 
El testimonio expresa la clara necesidad de delimitar demandas indígenas al interior del espacio político barrial, siendo ello un factor clave para dinamizar el proceso de organización etnopolítica. La experiencia de haber recorrido los barrios, hablado con vecinos sobre necesidades $y$ participado en reuniones junto con dirigentes $y$ funcionarios municipales, fortaleció y legitimó una práctica etnopolítica en Almirante Brown.

Aunque inicialmente dicha práctica estaba direccionada hacia el fortalecimiento cultural de una masa de migrantes indígenas en la periferia, esta se encontraba subsumida bajo modalidades partidarias y clientelares que desde el Partido Justicialista ${ }^{9}$ reproducía un grupo de referentes barriales. Es decir, al hacer nuevamente foco en el testimonio citado, la formación de una dirigencia indígena en Almirante Brown estuvo nutrida por aquellas relaciones interpersonales que el tejido barrial imprimió en sus trayectorias (Engelman, 2017).

El motivo de generar un espacio etnopolítico y de fomentar la participación indígena en el gobierno local, partía desde una ausencia y no desde una presencia. Es decir que el acceso al conjunto de políticas y programas estatales de la población indígena no se canalizó estatalmente a partir de la elaboración de políticas culturales, sino que se dio por la condición de vulnerabilidad socioeconómica.

Entre los diferentes programas de empleo directo o transitorio que se implementaron desde el gobierno nacional, se pueden mencionar el Programa Trabajar I y II de la década de 1990, y su posterior unificación en el año 2002 con la creación del Programa Jefes y Jefas de Hogar Desocupados. Luego se sumaron dos programas complementarios — creados en el año 2003-, siendo el primero, el Plan Mayores y el segundo el Programa de Empleo Comunitario (Manzano, 2013).

Al tiempo que este tipo de beneficios arribaban a la población urbana de Almirante

$9 \quad$ El Partido Justicialista (PJ) es un partido político argentino que continuó con las líneas del Partido Peronista. Así, este es una prolongación de la justicia social y está altamente vinculado a la defensa de los sectores populares y obreros.
Brown, las y los dirigentes indígenas llevaban a cabo tareas sociales a través de la formación de centros y salones comunitarios, del trabajo en sus viviendas - como fue el caso de la comunidad "Cacique Hipólito Yumbay"-, o mediante la labor que encaraban las mujeres en comedores a partir de las ayudas obtenidas, tanto por donaciones locales como las entregadas por el municipio. Esas tareas eran complementadas junto a la realización semanal de copas de leche $y$ placares comunitarios ${ }^{10}$.

Evidentemente, el entramado de vínculos barriales $y$ la heterogeneidad de sujetos involucrados reforzaron la autoadscripción local en un contexto material caracterizado por el incremento de programas y políticas sociales. En otras palabras, la ausencia de políticas o programas específicos para los indígenas en los barrios de la RMBA incentivó su organización y definió objetivos comunes en términos culturales. El primero de ellos buscó revertir aquella experiencia política partidaria por una de tipo etnopolítica, para definir estrategias y poder responder sobre algunas demandas y condiciones de vida de la población indígena.

En segundo lugar, esa reorientación se nutrió por una toma de conciencia acerca de su origen étnico gracias al fortalecimiento comunitario, como consecuencia de la visibilización y organización de la población indígena urbana. Este segundo punto es más aprehensible si se consideran los efectos positivos del reconocimiento constitucional del año 1994 y de la ratificación del Convenio 169 de la Organización Internacional del Trabajo (OIT) en el año 2001.

Por otro lado, ese marco de derechos será base para que la población indígena urbana dispute su participación al interior de los programas y políticas venideras, tanto a nivel municipal, provincial como nacional. El acta

10 Las copas de leche consistían en la organización semanal de una merienda que dirigentes realizaban a fin de garantizar el consumo de ciertos alimentos a niñas y niños de las comunidades. Asimismo, los placares comunitarios estaban asociados a labores de recolección y arreglo de prendas de vestir que, posteriormente, se redistribuían entre los miembros de las familias indígenas $y$ vecinos. 
de constitución del CIAB es expresión de la consolidación del espacio etnopolítico y enseña un clima de época respecto de las demandas indígenas contemporáneas:

Se reúnen representantes indígenas de distintas comunidades (...) para acordar la creación del Consejo Indígena de Almirante Brown, con el objeto y en el marco de nuestros derechos indígenas consagrados en el art. 75 inc. 17, las leyes 23.302 y 24.071, el Convenio 169 y el art. 36, inc. 9 (...) y acuerdan los siguientes puntos: a) participación de la política pública en el ámbito de Almirante Brown; b) cumplimiento de las normativas vigentes Nacional e Internacional; $y$ c) promover la unidad, organización y el progreso de los pueblos indígenas (Libro de Actas del CIAB, 29/04/2007, pp. 1-2).

El proceso de organización etnopolítico local, a diferencia de la década de 1990, se encauzará mediante demandas de participación étnica-ciudadana (Bengoa, 2009) y de un alto nivel de institucionalización de la práctica etnopolítica, como enseña el fragmento citado.

La relevancia de dicha institucionalización encuentra sentido si se considera que en los últimos 15 años, el número de comunidades reconocidas por el Estado Nacional y Provincial se incrementó. Almirante Brown en la actualidad posee 8 comunidades indígenas registradas, de las cuales 4 están inscriptas con personería jurídica del INAI y las demás se encuentran en trámite. Cabe mencionar que la octava comunidad, de las 7 mencionadas en el cuadro 1, es la "Willi Che", la cual se destaca por ser la única de carácter multiétnico, ya que reúne familias migrantes del pueblo Toba(Qom), Guaraní, Mapuche $y$ Ranquel ${ }^{11}$.

11 De acuerdo a la información que brindó el Consejo Provincial de Asuntos Indígenas de Buenos Aires, en la RMBA entre asociaciones y comunidades indígenas hay registrados unos 60 casos hasta el 2017. Si bien, este número se restringe solamente a aquellos nucleamientos indígenas que iniciaron el trámite para su reconocimiento estatal, Almirante
Hasta aquí se ha puntualizado acerca del conjunto de condiciones que permitieron dinamizar una coyuntura de organización etnopolítica en Almirante Brown. En cuanto a sus antecedentes, se entiende que el objetivo primordial fue la definición de un espacio de participación propio en el marco de derechos que reconocen a la población indígena. Esa estrategia de diferenciación dinamizó un proceso de autoadscripción de la población local muchas veces motivado por el posible manejo de recursos que el Estado destinaba a los barrios a través de diferentes programas y políticas sociales.

A continuación, se hará referencia a una experiencia transitada por un sector de la dirigencia indígena de Almirante Brown. Se trata de la participación de diversas comunidades en el denominado "Programa Inserción, Capacitación y Promoción de Derechos vinculados a Pueblos Originarios", que se llevó a cabo durante el año 2015 y fue implementado por la Defensoría del Pueblo de la provincia de Buenos Aires $^{12}$.

La elección y descripción de la mencionada experiencia, por un lado, resume el carácter contradictorio y externo que los programas estatales poseen respecto de la participación de las poblaciones indígenas, tanto en la consulta como en la toma de decisiones. Por otro lado, su corta duración permite destacar el carácter errático desde el cual muchas veces este tipo de programas funcionan, así como, su vulnerabilidad al interior de la estructura administrativa estatal. No obstante, a través de su ejemplificación se busca destacar aquellas instancias en que los y las dirigentes indígenas revierten la posición de subordinación a partir de procesos de negociación y resistencia local.

Brown concentra casi el 14\% de las comunidades de toda la región, precedido por la ciudad de La Plata con el casi 19\%.

12 La Defensoría del Pueblo de la provincia de Buenos Aires es un órgano de garantía, unipersonal, autónoma e independiente que promueve los derechos colectivos e individuales de los habitantes de la provincia y de aquellos que se encuentren en tránsito por esta. 
LA APLICACIÓN DEL "PROGRAMA INSERCIÓN, CAPACITACIÓN Y PROMOCIÓN DE DERECHOS VINCULADOS A PUEBLOS ORIGINARIOS" EN ALMIRANTE BROWN

En el año 2014, el "Programa de Inserción, Capacitación y Promoción de Derechos vinculados a Pueblos Originarios" (PICPDPO) inicia sus actividades como parte de una política de la Defensoría del Pueblo de la provincia de Buenos Aires. El equipo de trabajo que llevó adelante las tareas, estuvo integrado por 3 técnicos: un militante de una organización social, una abogada y una antropóloga. Como objetivo principal, el programa buscaba "regular, normativizar y replicar el reconocimiento $y$ protección de los derechos individuales y colectivos de las comunidades, así como la promoción y visibilización dentro del ámbito de la provincia de Buenos Aires" (Reglamento PICPDPO, 2014, p.1). Cabe mencionar, que el acercamiento inicial entre el equipo técnico y el CIAB fue en el mes de septiembre del año 2014, en el marco de una charla que diversos dirigentes indígenas realizaron en el Museo Etnográfico de la CABA. Finalmente, en el mes de noviembre ambas partes firmaron un acuerdo de trabajo colectivo para llevar adelante un "informe de situación de las comunidades originarias de Almirante Brown", como así también acompañar el reclamo territorial de un predio de 50 hectáreas efectuado por una de las comunidades ante el INAI.

De acuerdo a los testimonios etnográfi$\cos y$ a diferencia del proceso de organización etnopolítico del CIAB, el PICPDPO recorrería un camino contrario. Es decir, no fue un espacio creado por la población indígena, sino que fue el resultado de una política provincial que, según las opiniones de las y los dirigentes locales, "necesitó buscar a los indígenas de Buenos Aires para su implementación" (mujer dirigente Mocoví de "Nogoyin Ni Nala", localidad de Calzada, comunicación personal, 16/07/2015). En este sentido, se trata de pensar que el programa o las diversas áreas donde se llevan a cabo políticas destinadas a la población indígena, dependen de convenios y voluntades políticas inestables como de presupuestos reducidos. Esa situación no solo afectó la composición y las posibilidades del equipo técnico ${ }^{13}$, sino que este último muchas veces reveló, a través de diferentes conflictos, un conocimiento incipiente sobre los reclamos territoriales y demandas culturales de las poblaciones indígenas de la RMBA.

Ahora bien, el convenio de trabajo que la dirigencia indígena de Almirante Brown firmó con las personas técnicas provinciales permitió la realización de reuniones en la sede que la defensoría posee en la ciudad de La Plata, en los terrenos comunitarios o en encuentros organizados por los dirigentes indígenas en clubes sociales. El programa no se vinculó solamente con la dirigencia indígena del CIAB, sino que a lo largo del año 2015, se desarrollaron en total 18 actividades con comunidades ubicadas en los partidos de San Miguel, Moreno, Derqui, Escobar y José C. Paz de la RMBA (ver mapa 1) o en localidades como Los Toldos del interior de la provincia de Buenos Aires.

Este campo de acción provincial que se extendió en la medida en que las personas integrantes del equipo de trabajo, entraron en contacto con una diversa dirigencia, expresó el desconocimiento acerca de que muchas comunidades de la provincia de Buenos Aires contaban con una alta experiencia de organización etnopolítica ${ }^{14}$.

El equipo técnico buscaba fomentar un trabajo que permitiera ampliar las posibilidades laborales de las comunidades para que "los mismos indígenas", tal como ellos afirmaban, sean

$13 \quad$ El programa inició a finales de 2014, se desarrolló durante todo el 2015 y finalizó el primer trimestre de 2016. El equipo de trabajo estuvo constituido inicialmente por un funcionario estatal, una abogada y una antropóloga. Posteriormente, gracias a un conflicto interno, su composición fue modificada y la abogada $y$ antropóloga fueron reemplazadas por una arquitecta.

14 Para el Sur de la provincia de Buenos Aires, Isabel Hernández (1993) e Ingrid de Jong (1994 y 2004) desde la perspectiva de la Antropología Social analizaron la consolidación y organización de la población mapuche en la localidad de Los Toldos desde el período de la "Conquista del Desierto" hasta la actualidad; o desde la Antropología Histórica, Nora Guerci (2002) describe cómo se constituyó recientemente la "Asociación Peñi Mapu" en las localidades de Olavarría y Azul. 
quienes transformaran sus condiciones de vida. No obstante, aunque el optimismo que presentaban los técnicos y técnicas era incuestionable, su presupuesto era escaso. En consecuencia, en los diferentes encuentros se enfatizó que se buscaba "articular" y "vincular" a las comunidades con programas del Ministerio de Desarrollo Social y de Trabajo de la Nación ${ }^{15}$.

\section{LA MEDIACIÓN INSTITUCIONAL DE LA DEFENSORÍA DEL PUEBLO DE LA PROVINCIA DE BUENOS AIRES}

Vale detenerse en el carácter paradójico de las estrategias que el PICPDPO presentó a las personas dirigentes de Almirante Brown al elaborar y completar las planillas para la presentación de los proyectos. En primer lugar, según los funcionarios y funcionarias, la idea de entregar las propuestas en ambas dependencias "ampliaba" las posibilidades de obtener el beneficio. Según sus testimonios, eso además era posible gracias a la existente conexión que había entre ellos con los técnicos y técnicas de ambos ministerios nacionales. En segundo lugar, otra de las estrategias recomendadas fue que la posibilidad de presentarse al programa sería solamente para aquellos que cumplieran con los requisitos formales ${ }^{16}$, y que posteriormente podían ser incorporados los demás integrantes comunitarios. Y en tercer lugar, las planillas y las actividades a realizar debían ser elaboradas desde "una estrategia que priorizara lo tradicional y cultural". Este último punto, además de ser el más controversial, generó un descontento generalizado en las personas dirigentes y en los presentes de la reunión:
¿Acaso vos nos ves haciendo cuenquitos de cerámica o tejiendo cestas? Nosotros $y$ ellos [señalando a los jóvenes presentes en el comedor] necesitamos formación, capacitarnos para poder manejar computadoras, internet $y$ todo eso. Ahora sí o sí te piden que tengas esos conocimientos como básicos para cualquier trabajo (mujer Dirigente Mocoví de "Nogoyin Ni Nala", localidad de Calzada, comunicación personal, 16/07/2015).

Es importante señalar que uno de los proyectos a presentar era de fabricación de ladrillos de arcilla - para los hombres-y el otro de artesanías - para las mujeres-. En ningún momento, las personas funcionarias provinciales se cuestionaron acerca del sentido que ellos y ellas tenían sobre sus representaciones de la población indígena de la RMBA. Al respecto, Liliana Tamagno (1991) manifiesta que durante las situaciones de reparación de derechos entre el Estado y las poblaciones indígenas- se erigen un conjunto de "censores de indianidad" que juzgan desde criterios propios y no discutidos con el grupo en cuestión, la pertinencia o no del reclamo. A fin de graficar el sentido ahistórico y esencializador que los funcionarios $y$ las funcionarias del programa reprodujeron, se adjunta el siguiente folleto difundido por la Defensoría del Pueblo de la provincia de Buenos Aires (imagen 1).
15 Uno de los proyectos se llamaba "Manos a la Obra" y formaba parte del programa "Bases Firmes" del Ministerio de Desarrollo Social. En el caso del Ministerio de Trabajo, las presentaciones se harían a través del programa "Trabajo Autogestionado".

16 El equipo de trabajo que presentaba el proyecto debía estar desocupado. No podía tener un empleo formal y tampoco debía percibir ayudas o beneficios estatales. 
IMAGEN 1

PORTADA DEL FOLLETO ELABORADO POR LA DEFENSORÍA DEL PUEBLO DE LA PROVINCIA DE BUENOS AIRES PARA PROMOCIONAR EL ÁREA DE PUEBLOS ORIGINARIOS Y EL "PROGRAMA

INSERCIÓN Y CAPACITACIÓN Y PROMOCIÓN

DE DERECHOS VINCULADOS A PUEBLOS ORIGINARIOS"

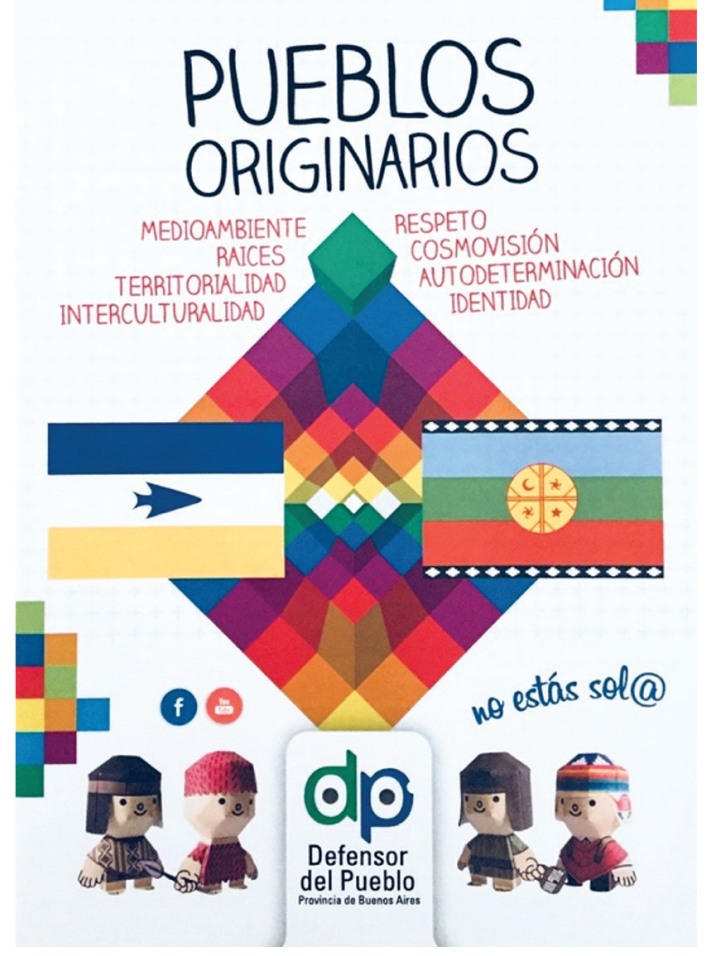

Fuente: Fotografía tomada por el autor, 2016.

La imagen y la definición acerca de "lo indígena" limitaba las posibilidades reales de presentar proyectos vinculados a las necesidades y problemáticas de las condiciones de vida urbana. Sus reclamos de clase se veían invalidados por una presentación de proyectos que enfatizaba una representación folklórica de su identidad. Por ejemplo, algunos y algunas dirigentes habían llevado propuestas para desarrollar pequeños emprendimientos colectivos (panaderías, textiles, peluquería), que al tiempo que beneficiaban la inserción laboral ponderaban los oficios y los conocimientos de los que disponían. Sin embargo, ese encapsulamiento no invalidó que los y las dirigentes del CIAB, además de presentar los proyectos sugeridos por el PICPDPO, lo hicieran de forma autónoma $y$ directa ante los ministerios correspondientes.

Esto último fue posible gracias a dos situaciones. La primera de ellas tuvo que ver con la capitalización y socialización que esta capa de hombres y mujeres dirigentes urbanos aprehendió a través de la experiencia política barrial de décadas pasadas, y su posterior organización etnopolítica en el espacio local. Es aquí donde los diferentes programas de empleo directo o transitorio que se implementaron desde el gobierno nacional y que fueron mencionados anteriormente, tomaron relevancia. Las y los dirigentes, así como, las familias indígenas no solo accedieron a diversos planes y programas de ayuda social, sino que también fueron protagonistas en su redistribución. Este tipo de experiencias, posibilitó que un sector de la población indígena de Almirante Brown se legitimara al interior del espacio etnopolítico y barrial, manifestando un mayor grado de autonomía y empoderamiento ${ }^{17}$ en la búsqueda de recursos estatales. El siguiente testimonio, que también pertenece a una reunión entre la dirigencia del CIAB $y$ las personas funcionarias del programa provincial, es prueba de ello:

No nos pueden decir qué tenemos que hacer, y cómo hacerlo. Acá no hubo consulta, se mandaron solos (...). Nosotros nos vamos a manejar con los Ministerios como lo hicimos la vez que pudimos tener los proyectos productivos (hombre dirigente Kolla de "Guaguajni Jallpa", localidad de Glew, comunicación personal, 16/07/2015).

Si la formación etnopolítica y barrial de la década de 1990 fue la primer condición que

17 La noción de empoderamiento o de "empowerment" ha sido recientemente retomada por las ciencias sociales. Aunque refiere a procesos que no son novedosos, su aplicación se destaca para abordar el conjunto de condiciones acerca de la organización actual de colectivos de género o grupos étnicos como el caso de los afrodescendientes. De acuerdo con Betancor (2011), la noción surge del aportes de Paulo Freire y sus "campañas de alfabetización” y de la obra de Solomón. 
enseña el grado de autonomía y empoderamiento de la población indígena respecto de áreas estatales, el segundo punto a destacar tiene que ver con la relevancia que las relaciones de parentesco $y$ alianza ${ }^{18}$ poseen en el tejido urbano.

Interesa destacar que las relaciones que dinamizan los procesos de organización etnopolítica contemporánea están fuertemente vinculados a una distribución regional de las familias indígenas que se condice con los procesos migratorios; aspecto que excede las áreas o jurisdicciones delimitadas por los municipios. Por ejemplo, en un encuentro sobre la problemática territorial - organizado en el marco del convenio de trabajo junto con el PICPDPOacudieron referentes indígenas de localidades, tales como: Tres de Febrero, Moreno, Quilmes, Don Orione, Lanus, Guernica, Calzada y Monte Grande (ver mapa 1). En primer lugar, el carácter regional evidencia una distribución de la población indígena en el tejido urbano que aparenta ser dispar. No obstante, si la mayoría de las y los dirigentes han compartido espacios de formación, protesta $y$ participación etnopolítica desde la década de 1980 en adelante, esa primera impresión es fácilmente revertida. Pero esto no sucede solamente si se consideran las experiencias y trayectorias pasadas, sino que también es posible hacerlo a través de la descripción de las demandas actuales.

$\mathrm{El}$ encuentro al que se hace referencia se llevó a cabo en el año 2015, en el cual se expuso el compromiso acaecido entre el CIAB y los técnicos y técnicas provinciales acerca de un reclamo territorial de 50 hectáreas en la localidad de $\mathrm{Glew}^{19}$. Las y los dirigentes indígenas

$\overline{18 \quad \text { En }}$ otras publicaciones se ha abordado sobre los procesos migratorios de las diversas comunidades y puntualizado acerca de la función receptora que caracteriza a las relaciones entre parientes durante los primeros años en que las familias indígenas arribaron a la RMBA (Engelman y Weiss, 2015 y Engelman, 2016).

19 En el año 2006, el Poder Legislativo Nacional sancionó y declaró la "Ley de Emergencia en materia de Posesión y Propiedad de las Tierras Comunitarias" nro. 26.160. Dicha ley busca resguardar los territorios que las poblaciones originarias habitan tradicionalmente. Es bajo ese marco jurídico que la comunidad "Guaguajni Jallpa" de expusieron la importancia que dichas tierras poseen tanto para mejorar las condiciones de hacinamiento de las familias como de las potencialidades productivas ${ }^{20}$ que en estas podrían llevarse a cabo. En su mayoría, relataron que el incremento demográfico se debe tanto al crecimiento natural de las familias, como a la migración de parientes que arriban a la RMBA en busca de trabajo ${ }^{21}$.

En segundo lugar, otra problemática que fue expresada en el encuentro fue la escasez de tierras en relación al crecimiento que protagonizó la periferia urbana, y cómo todo ello afecta al generar problemáticas entre vecinos y al interior de las familias indígenas - tomas de tierras, violencia directa, discusiones intervecinales, robos, etc.-. A su vez, ese crecimiento poblacional no se condice con la extensión de servicios públicos, tales como, el agua potable, el gas o la electricidad. Esto aún se complejiza más, si se considera que algunas familias indígenas viven en pequeñas casas de material o de madera, construidas sobre terrenos ocupados de difícil acceso, dada la falta de calles en buen estado o de transporte público. Otro eje en que varios dirigentes coincidieron fue en la necesidad de compartir el espacio, y sobre el uso colectivo que tendría el predio reclamado en caso de ser obtenido.

Este reclamo territorial no es solo para las familias Kollas, sino para las familias Qom, Mocoví, Guaraní, Diaguita. Todos tenemos problemas con el espacio. Muchas familias viven todas juntas en

Glew lleva a cabo el reclamo de 50 hectáreas, relevadas por el INAI en el año 2009.

20 Entre estas se pueden mencionar la realización de huertas comunitarias, la construcción de espacios comunes para el desarrollo de talleres de capacitación o de comedores, así como de micro emprendimientos productivos, tales como, panaderías, herrerías, etc.

21 Las familias indígenas asentadas en el RMBA están fuertemente vinculadas con parientes del interior del país y de sus lugares de procedencia. Las relaciones existentes entre "lo rural" y "la ciudad" tensionan esas categorizaciones en una coyuntura que se caracteriza por una dinámica constante de flujos de personas, información y comunicación. 
una misma casa, en una misma habitación y eso trae problemas (hombre dirigente Kolla de "Guaguajni Jallpa", localidad de Glew, comunicación personal, 02/02/2015).

En términos generales, el conjunto de demandas que se vinculan con el reclamo territorial están representadas al inicio del trabajo. Como se señaló anteriormente, los datos que arrojó el INDEC sobre vivienda están fuertemente relevados en las descripciones etnográficas de la dirigencia indígena. Lo mismo sucedió con los datos referidos al trabajo si se considera que en los últimos años la importancia de los programas y políticas sociales para las familias indígenas se ha incrementado, dado que su percepción representa un alto porcentaje de sus ingresos mensuales.

Sin embargo, sería erróneo reparar únicamente sobre el impacto material que este tipo de ayudas sociales conllevan, si bien son base para la reproducción económica de las familias indígenas, su manipulación y manejo - al interior de las relaciones interculturales - permitieron reorientar y significar nuevas demandas etnopolíticas en la RMBA.

Por otro lado, la búsqueda de escindir las trayectorias de formación política barrial y constituirse como espacio de organización propio y etnopolítico, permite entender la reorientación de objetivos de la dirigencia indígena de Almirante Brown. De modo que la participación en espacios estatales se ha naturalizado como el horizonte de los nuevos reclamos, siendo el marco para, posteriormente, definir políticas públicas indígenas-ciudadanas (Bengoa, 2009).

Si se considera que no participaba ningún dirigente indígena al interior del equipo de trabajo del PICPDPO, no resulta extraño que quienes encabezaban el CIAB deslegitimaran el rol de la población funcionaria y del organismo provincial. Esa falta de participación en el programa, fue eje para reclamar la incorporación de un o una dirigente indígena y revertir dicha falencia. A fines del año 2015, es cuando finalmente se logra la contratación de un o una dirigente - elegido o elegida por asamblea-que llevaría adelante la participación y consulta que ratifican los convenios internacionales $y$ derechos contemporáneos

Por último, cabe mencionar que los resultados obtenidos por el PICPDPO no resolvieron ni las demandas territoriales ni aquellas vinculadas a las condiciones de vida de las familias indígenas de Almirante Brown. Además, quien fue elegido para saldar la falta de participación indígena en el programa, al ser cerrado, este nunca asumió. La transición y paso del gobierno de la República Argentina de la Dra. Cristina Fernández de Kirchner (en los periodos electorales que van desde el año 2007-2011 y 2011-2015) al actual gobierno del Ing. Mauricio Macri (electo presidente en el año 2015), implicó el cierre de varios programas destinados a las poblaciones indígenas, dada la implementación de un modelo neoliberal cuyo eje es el recorte del gasto público en pos de solicitar capitales y deuda extranjera. Un ejemplo relevante ha sido el recorte sobre programas sociales en general y sobre la política territorial que el INAI desarrolló durante los años del 2000. Esto último se reflejó en el despido del $25 \%$ de su planta, lo que no solo ha reducido su alcance federal, sino sus capacidades efectivas en torno a la resolución de conflictos y problemáticas indígenas (Trinchero et ál., 2018).

\section{REFLEXIONES FINALES}

En la actualidad, según palabras de Miguel Bartolomé, se enfrentan procesos que se podrían considerar de reetnización (2010). Es decir, una coyuntura derivada de experiencias de participación política de décadas pasadas que, gracias a la influencia de las organizaciones etnopolíticas, contribuyeron a dignificar lo étnico $y$ otorgarle un sentido positivo a la condición indígena en la Argentina. Además, en términos metodológicos, se entiende que retomar variables, tales como, el crecimiento demográfico o criterios censales para comprender la presencia indígena en la RMBA resulta insuficiente. Se busca problematizar aquellas posturas - o construcciones de sentido- que explican la presencia indígena en las ciudades como un fenómeno novedoso; cuando, en realidad, la emergencia de nuevas o viejas identidades propone nuevos contenidos que 
reconfiguran las designaciones étnicas en que fueron constituidas históricamente.

En estos casos las identificaciones no se "inventan" sino que se actualizan, aunque esa actualización no recurra necesariamente a un ya inexistente modelo prehispánico. Se trata de recuperar un pasado propio, o asumido como propio, para reconstruir una membresía comunitaria que permita un acceso más digno al presente (Bartolomé, 2010, p.14).

El empoderamiento de la población indígena de Almirante Brown puede ser comprendido de este modo, como el resultado de experiencias compartidas de organización etnopolítica altamente dinamizadas por relaciones de parentesco y alianza local.

Ese tejido, como se ha señalado, al tiempo que se nutrió de relaciones interculturales —de militancia y política barrial- habilitó la ejecución de demandas de participación a través de mayores canales de diálogo y autonomía. Ello quedó claro mediante la consolidación de una dirigencia indígena local entre la década de 1990 e inicios del 2000, que logró constituirse como "Consejo Indígena" y que más allá de su composición multiétnica delineó un plan de trabajo en común.

De hecho, aunque se ha reparado acerca de que la heterogeneidad es una de las características que define a la población indígena urbana, se puede sostener que esta no inhibe su organización etnopolítica.

En un trabajo reciente, Araceli Burguete Cal y Mayor sostiene que los gobiernos locales - municipios - son instancias de gobernanza que los pueblos indígenas conocen históricamente, y que ello ha provocado una lucha constante que tiene por objetivo (re)significar o etnizar las instituciones del Estado (2011). Se entiende que resaltar esa dinámica de lucha constante, permite abordar críticamente aquella posición de subordinación con que muchos trabajos - a veces a priori- abordan las modalidades de negociación y participación contemporánea de las poblaciones indígenas y las instituciones del Estado.
En esta línea, Alcida Rita Ramos (2011) expone que ha sido fundamental el modelo de fricción interétnica en la antropología brasileña, y que si bien, este continúa dando cuenta de una realidad desigual y conflictiva entre la población indígena y la civil, reconoce que el papel político de los primeros se modificó fundamentalmente en las últimas décadas.

Otra prueba de esto ha sido cuando la dirigencia indígena de Almirante Brown expresó conscientemente que el equipo de trabajo del PICPDPO "necesitó buscar a los indígenas de la provincia de Buenos Aires" para su funcionamiento. Si bien, no se niega la existencia de una discriminación política, muchas veces traducida en cuestionar $y$ deslegitimar la identidad de los indígenas en la ciudad (Engelman, 2016), se entiende que la reetnización ha dado como consecuencia resultados positivos y tangibles en los procesos de negociación interétnica respecto a décadas pasadas ${ }^{22}$.

Finalmente, cabe mencionar que las posibilidades de negociación y participación indígena se han visto comprometidas desde el año 2015, cuando el bloque político "Cambiemos", encabezado por Mauricio Macri, asume la presidencia. En términos generales, la reducción fiscal a través del achicamiento de las funciones estatales, aplicado por el nuevo gobierno, derivó en el cierre de programas y beneficios sociales, lo que rápidamente se tradujo en el desmejoramiento de las condiciones de vida de la población vulnerable, entre ellas, la indígena, quienes frente a un alto proceso inflacionario debieron restringir su capacidad de consumo.

En el caso del Almirante Brown, esto impactó por ejemplo en la organización de comedores sociales, en la imposibilidad de realizar "eventos culturales" u otros mediante la expresión de un trabajo etnopolítico focalizado al espacio local o barrial.

Aunque el presente trabajo no tuvo por objetivo presentar la coyuntura actual, sí se

22 Más allá de que no se hizo efectiva la incorporación de un dirigente indígena al equipo de trabajo de la Defensoría del Pueblo de la provincia de Buenos Aires, los recursos estatales para que el cargo existiese, fueron conquistados gracias a las demandas etnopolíticas. 
considera importante analizar en futuras producciones el impacto que las mencionadas transformaciones de las condiciones de vida poseen tanto en la formación de demandas indígenas como en su práctica etnopolítica. A decir verdad, se espera que la capitalización de experiencias de las recientes dos décadas pasadas sea base suficiente para que tanto las dirigencias indígenas como sus familias, puedan revertir su posición de subordinación mediante el rol activo que han venido adquiriendo recientemente.

\section{REFERENCIAS}

Amodio, E. (1996). Los indios metropolitanos: Identidad étnica, estrategias políticas y globalización entre los pueblos indígenas de América Latina. En D. Mato, M. Montero y E. Amodo (eds.), América Latina en tiempos de globalización: procesos culturales y transformaciones sociopoliticas (pp. 51-66). Caracas, Venezuela: UCV-ALAS-UNESCO.

Balazote, A. (2015). Pueblos Originarios: disputas en el campo discursivo. Revista GeoPantanal, 18, 33-50.

Bartolomé, M. (2010). Interculturalidad y territorialidades confrontadas en América Latina. RUNA, 31, 9-29.

Bengoa, J. (2009). ¿Una segunda etapa de la Emergencia Indígena en América Latina? Cuadernos de Antropología Social, 29, 7-22.

Betancor, M. (2011). Empoderamiento: ¿una alternativa emancipatoria? Revista Margen, 61, 1-14.

Burguete Cal y Mayor, A. (2011). Municipalización del gobierno indígena e indianización del gobierno municipal en América Latina. Revista Pueblos y Fronteras Digital, 11, 38-88.

De Jong, I. (2004). De la asimilación a la resistencia: disputas en torno al pasado entre la población indígena de Los Toldos (provincia de Buenos Aires). Cuadernos de Antropología Social, 20, 131-151.

Engelman, J. (2016). Migración étnica y condiciones de vida urbana al sur del
Área Metropolitana de Buenos Aires. Alteridades, 26, 67-79.

Engelman, J. (2017). Clientelismo y etnicidad: el proceso de formación del "Consejo Indígena de Almirante Brown" en provincia de Buenos Aires, Argentina. Revista Antropologías del Sur, 7, 39-54.

Engelman, J., y Weiss, M. L. (2015). El imán de la ciudad: migración y distribución espacial de población indígena en el Área Metropolitana de Buenos Aires, Argentina. Revista Geopantanal, 18, 51-70.

Guerci, N. (2002). La asociación Peñi Mapu: conformación de una identidad étnica en contextos de globalización. Actas del 3er Congreso Virtual de Antropología y Arqueología. Recuperado de http://www. equiponaya.com.ar/congreso2002/ponencias/marcela_guerci.htm

Hernández, I. (comp) (1993). La Identidad Enmascarada. Los mapuche de Los Toldos. Buenos Aires, Argentina: Eudeba. INDEC (2012). Censo Nacional de Población, Hogares y Viviendas. 2010. Censo del Bicentenario. Resultados Definitivos, Serie B, nro. 2, Tomo I. Buenos Aires, Argentina.

INDEC (2015). Censo Nacional de Población, Hogares y Viviendas. 2010. Censo del Bicentenario. Pueblos Originarios, Región Metropolitano. Serie D, nro. 6. Buenos Aires, Argentina.

Libro de Actas. (2007). Actas del Consejo Indígena de Almirante Brown.

Maceira, V. (2002). Notas para una caracterización del Área Metropolitana de Buenos Aires. Universidad Nacional de General Sarmiento, Argentina. Recuperado de http://www.observatoriodasmetropoles. net/download/notas_regi\%C3\%B3n_ metropolitana_de_Buenos\%20Aires.pdf

Maidana, C. (2009). Volver a la tierra. Parentesco, redimensionalización territorial y reconstrucción identitaria. En L. Tamagno (coord.). Pueblos Indígenas: Interculturalidad, colonialidad, politica (pp.45-58). Buenos Aires, Argentina: Editorial Biblos. 
Manzano, V. (2013). La política en movimiento. Movilizaciones colectivas y políticas estatales en la vida del Gran Buenos Aires. Rosario, Argentina: Protohistoria Ediciones.

Programa Inserción, Capacitación y Promoción de Derechos vinculados a Pueblos Originarios (2014). Reglamento.

Pérez Ruiz, M. (2008). Diversidad, identidad y globalización. Los jóvenes indígenas en las ciudades de México. En M. L. Pérez Ruiz (Coord). Jóvenes indígenas y globalización en América Latina (pp. 45-68). Ciudad de México, México: INAH.

Ramos, A. (2011). Por una antropología ecuménica. En A. Grimson, S. Merenson y G. Noel (comp). Antropología Ahora. Debates sobre la alteridad (pp. 97-124). Buenos Aires, Argentina: Siglo XxI.

Ratier, H. (1975). Villeros y Villas Miserias. Buenos Aires, Argentina: Centro Editor de América Latina.

Tamagno, L. (1991). La cuestión indígena en la Argentina y los censores de la indianidad. Revista América Indígena, 1, 123-152.
Tamagno, L. (2001). Nam Qom Hueta 'a Na dockshi Lma: Los tobas en la casa del hombre blanco. Identidad, memoria $y$ utopía. La Plata, Argentina: Ediciones al Margen.

Tamagno, L. (2014). Indígenas en la ciudad. Organización política en contextos de tensión entre patrimonio cultural y políticas públicas. En L. Tamagno y M. Maffia (Coords). Indigenas, africanos y afrodescendientes en la Argentina. Convergencias, divergencias y desafíos (pp. 43-62). Buenos Aires, Argentina: Editorial Biblos.

Trinchero, H. (2010). Los Pueblos Originarios en la formación de la Nación Argentina. Revista Espacios, 46, 106-123.

Trinchero, H., Balazote, A., Radovich, J. C., Castilla, M., Engelman, J., y Valverde, S. (2018). Pueblos Indígenas en Argentina: fronteras históricas y contemporáneas. Mesclando Fronteiras na construçao dos Direitos Humanos (en prensa).

Fecha de ingreso: 08/11/2017 Fecha de aprobación: 02/10/2018 
